\title{
Self-organized pacemakers and bistability of pulses in an excitable medium
}

\author{
Michael Stich* \\ Centro de Astrobiología (CSIC/INTA), Ctra de Ajalvir km. 4, 28850 Torrejón de Ardoz (Madrid), Spain
}

Alexander S. Mikhailov

Abteilung Physikalische Chemie, Fritz-Haber-Institut der Max-Planck-Gesellschaft, Faradayweg 4-6, 14195 Berlin, Germany

Yoshiki Kuramoto

Research Institute for Mathematical Sciences, Kyoto University, Kyoto 606-8502, Japan

(Received 6 October 2008; published 23 February 2009)

\begin{abstract}
Pattern formation in an excitable medium described by a three-component reaction-diffusion system is investigated. Our focus is on stable self-organized pacemakers which give rise to spatially extended target patterns. Bistability of pulse solutions in the excitable regime is also reported, and interactions of the different pulses with each other and the pacemaker are studied. Self-organized pacemakers are created by a suitable perturbation from the steady state or through interaction of pulses. Bound states of one-dimensional pacemakers and phase flips are also observed.
\end{abstract}

DOI: 10.1103/PhysRevE.79.026110

PACS number(s): 82.40.Ck, 05.65.+b, 89.75.Kd

\section{INTRODUCTION}

Reaction-diffusion systems such as the BelousovZhabotinsky (BZ) reaction display a rich variety of spatiotemporal wave patterns $[1,2]$. The first wave pattern discovered in the $\mathrm{BZ}$ reaction consisted of concentric waves that were emitted periodically by a wave source [3]. The pattern formed by these waves is called a target pattern and the corresponding wave source a pacemaker. Target patterns of the same or similar nature have been observed in a variety of chemical, physical, and biological systems (see, e.g., [4-7]), which indicates that target patterns are generic for nonlinear dissipative media.

Due to the periodic feature of the pattern, target patterns are typically associated with oscillatory systems. A simple theoretical explanation of target patterns in oscillatory systems is that the pacemakers are created by heterogeneities which modify the properties of the medium such that the oscillation frequency is locally increased (see Ref. [8] and references therein). However, it is not necessary that the whole medium be oscillatory. It is sufficient that just a small part of the medium is oscillatory, while the rest of the medium is excitable. The oscillatory region creates waves that enter and propagate through the excitable region of the medium and form a target pattern. Also in this case it is assumed that heterogeneities are present, being capable of driving the medium locally into the oscillatory state.

However, experimental observations where pacemakers could not be related to any impurities in the BZ reaction [9] justify the general question of whether self-organized pacemakers, representing an intrinsic dynamical property, are possible in the absence of heterogeneities. Besides, target pattern formation in liquid crystals [5] or Dictyostelium discoideum colonies [10] has been directly related to the internal dynamics of the system. Another motivation of the study

\footnotetext{
*stichm@inta.es
}

of self-organized pacemakers comes from the theoretical side: while spiral wave patterns are stable solutions for many oscillatory and excitable two-component systems, selforganized target patterns seem to be unstable (at least if no additional coupling mechanism besides diffusion is considered). Therefore, three-component systems moved into the focus of attention (see, e.g., Refs. [11,12] and Ref. [13] for a model of the above-mentioned experiment [5]). Independently, the investigation of pattern formation in threecomponent reaction-diffusion systems has attracted growing interest in recent years; see, e.g., Refs. [14-18].

In this article we discuss a three-component reactiondiffusion system, introduced in Sec. II, which has stable solutions representing self-organized pacemakers in the excitable regime (Sec. III). We emphasize that, although stable self-organized pacemakers have been reported (see Ref. [8] and references therein) for oscillatory systems, the same does not hold for excitable media. Bistability of pulse solutions is also observed (Sec. IV), being an interesting and nontrivial pattern for reaction-diffusion systems. We compare the pulse solutions and target waves and describe the interaction of the different spatiotemporal solutions with each other (Sec. V). The article is closed with a discussion of the results (Sec. VI).

\section{MODEL}

The model investigated in this article is given by the reaction-diffusion system

$$
\begin{gathered}
\tau_{u} \partial_{t} u=u-u^{3}-v+l_{u}^{2} \nabla^{2} u, \\
\tau_{v} \partial_{t} v=\alpha u+\beta-v-\kappa(s-u), \\
\tau_{s} \partial_{t} s=u-s+l_{s}^{2} \nabla^{2} s,
\end{gathered}
$$

which describes the evolution of three variables $u, v$, and $s$ in spatially extended one- or two-dimensional domains. The 
parameters $l_{u}$ and $l_{s}$ represent the diffusion lengths and $\tau_{u}, \tau_{v}$, and $\tau_{s}$ the characteristic time scales of the system variables. The parameters $\alpha$ and $\beta$ describe the local kinetics of the variable $v$. The variable $s$ obeys simple linear kinetics and is coupled linearly to the variable $v$ via the parameter $\kappa$.

For $\kappa=0$, the model decouples and the subsystem (1a) and (1b) represents for $\alpha>0$ an activator-inhibitor system consisting of an activator $u$ and an inhibitor $v$. We choose $\alpha$ $=1, \beta=0.2, \tau_{u}=0.1$, and $\tau_{v}=1$ for all simulations shown in this article. For these parameters, the activator $u$ is fast compared to the inhibitor $v$ and the subsystem (1a) and (1b) is in the excitable regime: The only attractor is a fixed point, while an appropriate and sufficiently large perturbation of the stationary state leads to a large excursion in phase space before returning to it. The typical pattern appearing in a spatially extended excitable system is a pulse which propagates with constant shape and speed. After returning to its stationary state, the medium can be reexcited, thus supporting the propagation of pulse trains. The pulse solution does not depend on the specific details of the perturbation once the threshold is crossed. If a perturbation does not reach the critical magnitude and width, the perturbation directly decays to the steady state. For the stable pulse solution to exist, diffusion of $v$ is not essential and not assumed in this model.

The dynamics of the additional variable $s$ only depends on itself and the activator $u$; the inhibitor $v$ does not appear in Eq. (1c). Formally, the additional variable $s$ can be interpreted as either an activator or an inhibitor, depending on the value of the coupling coefficient $\kappa$. If $\kappa>0, s$ inhibits the inhibitor $v$, which is assumed below. Since the coupling term is proportional to the difference $s-u$, the coupling also modifies the activatory role of $u$. However, as long as $\kappa>-\alpha$ is fulfilled, the variable $u$ still behaves as an activator for $v$.

The set of equations (1) can be interpreted as a relatively simple extension of a standard activator-inhibitor model. We consider the case that the time scale of the variables $s$ and $u$ are of the same order and that $s$ diffuses more strongly than $u$. The coupling constant $\kappa$ usually takes positive values of order unity. Therefore, $s$ inhibits $v$ and mimicks the role of a second activator, which, however, diffuses more strongly than $u$.

For numerical simulations, we use explicit Euler and fourth-order Runge-Kutta schemes, three- and five-point representations of the Laplacian operators, and different scalings of the diffusion coefficients and system sizes, given in the respective figure captions.

\section{SELF-ORGANIZED PACEMAKERS IN THE EXCITABLE REGIME}

In this section, we investigate the formation of selforganized pacemakers in the excitable regime of the model (1). Setting $\alpha=1, \beta=0.2, \tau_{u}=0.1$, and $\tau_{v}=1$, the subsystem (1a) and (1b) is in the excitable regime. Choosing furthermore $\kappa=1.0$ and $\tau_{s}=0.1$, relaxational oscillations are absent for $\beta>0.143$, assuring that also the local dynamics of the full system is excitable. We assume that diffusion of $s$ is strong compared to diffusion of $u$. In such a system, the initial condition, such as the width of a superthreshold per- turbation of the rest state, determines whether the perturbation gives rise to a pulse pair or a pacemaker. This is shown in Fig. 1. On the one hand, a pair of pulses is created if the perturbation has a very large spatial extension [Figs. 1(a) and 1(c)]. On the other hand, if the width of the perturbation is small, a pacemaker is created [Figs. 1(b) and 1(d)]. The stable fixed point for these simulations is given by $u_{\mathrm{s}}=s_{\mathrm{s}}=-0.58$ and $v_{\mathrm{s}}=-0.38$. The thin solid lines in Figs. 1(a) and $1(\mathrm{~b})$ indicate the initial condition of $u$, where the perturbation consists of a constant shift to $u=0$ within a region of width $R$.

If the perturbation is wide, a pair of pulses is emitted. The thick lines in Fig. 1(a) denote the distributions of $u, v$, and $s$ shortly after the emission of the pair of pulses. The subsequent evolution in the central region is characterized by a decay to the stationary state, and the two pulses remain the only excited regions in the system. Note that the distribution of the activator $u$ possesses two local maxima at positions where the boundaries of the initial perturbation were located before. These maxima are associated with the nonmonotonous, oscillatory tail of each pulse and are typical for a pulse in an excitable regime close to the onset of oscillations. In the space-time diagram that shows the formation of a pulse pair [Fig. 1(c)], the local maxima of $u$ can be identified as dark lines in the middle of the displayed time interval. Later, these maxima subsequently develop to the first maxima in the oscillating tails of the pulses. The inhibitor $v$ also shows two maxima at these positions, which, however, are less pronounced. The component $s$, on the other hand, shows a broader profile and has its maximum in the center of the pattern. This is not surprising because the diffusion length $l_{s}$ is relatively large.

In Figs. 1(b) and 1(d), the same system is shown where the width of the initial perturbation of $u$ is smaller (but still large enough to trigger the formation of waves). After the emission of the first waves, the distribution of $u$ first also shows two maxima like those in Figs. 1(a) and 1(c). Then, these maxima merge in the central part of the pattern because they are located sufficiently close to each other. This local increase in the activator $u$ in the center constitutes a superthreshold perturbation which creates a new pair of waves. In terms of the variable $s$, we can describe the process in the following way: The component $s$ shows a broader profile due to its larger diffusion length and reaches its maximum value in the center of the pattern. Since $s$ decreases the value of the inhibitor $v$ wherever $s>u$ (and in this way acting as an activator), the accumulation of $s$ in the center with a sufficiently broad distribution facilitates the increase of $u$ in the center of the pattern. This scenario of wave emission is repeated, and a pacemaker is established. The stability of the pacemaker solution has been checked through long simulations which comprise thousands of wave emissions $(t=5000)$.

In order to understand the formation of stable selforganized pacemakers, we have varied the parameters $\beta, \kappa$, and $l_{s}$, using two different simulation protocols. First, we have applied variable widths of the initial perturbation of $u$, and second, we have started with a developed stable pacemaker for $\beta=0.2, \kappa=1.0$, and $l_{s}=1.0$ and changed the parameters in small steps. Of course, in the limit $\kappa \rightarrow 0$, the two- 

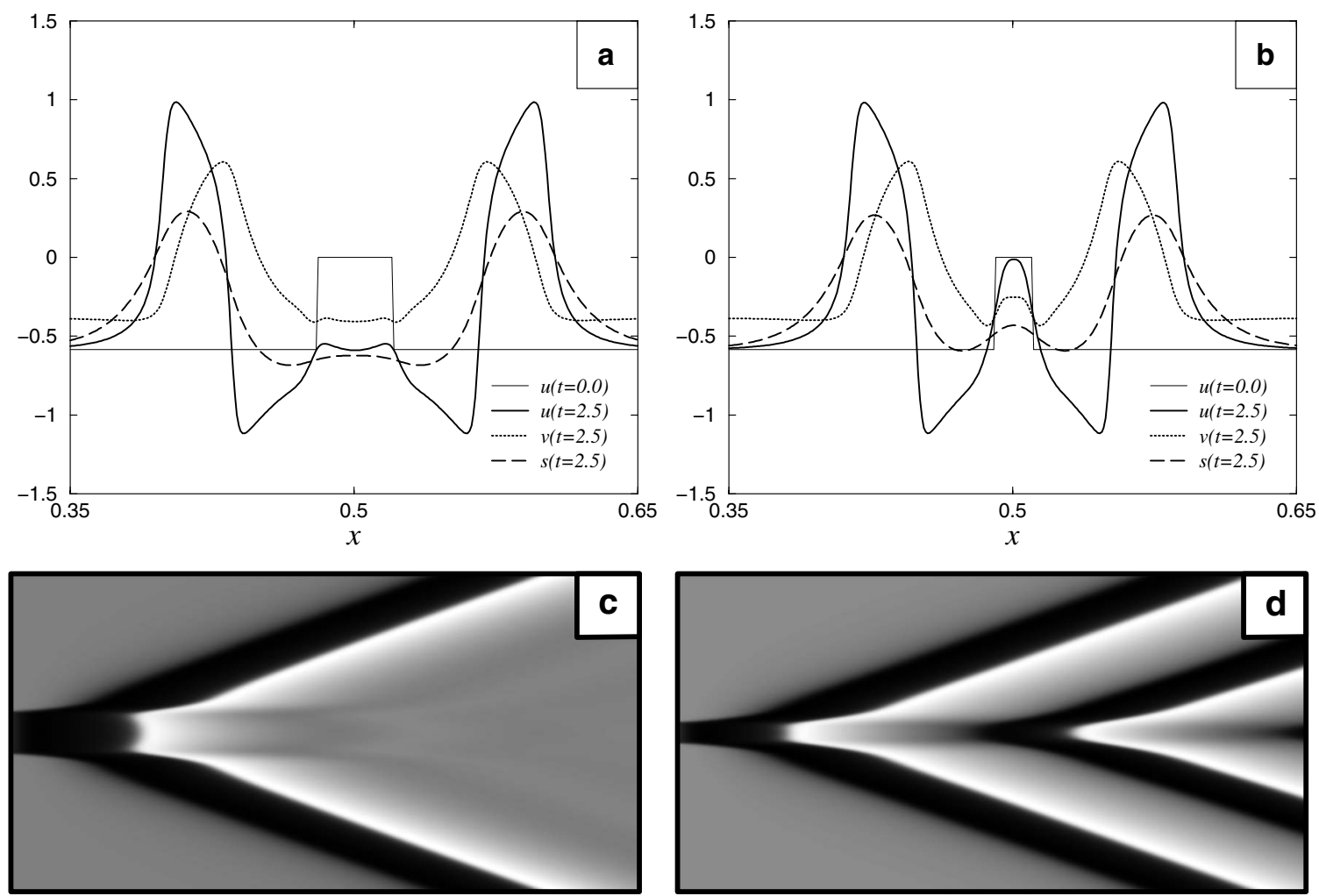

FIG. 1. Formation of pulse pairs and pacemakers. Characteristic stages in the development of a pulse pair (a) and a pacemaker (b) are displayed. The initial conditions for $u$ are shown as thin solid lines. Space-time diagrams for the formation of a pulse pair and a pacemaker are displayed in (c) and (d), respectively. There, the variable $u$ is shown in gray scale. The gray scale maps are always scaled to the minimum (white) and maximum (black) values of the variable in the displayed time interval. Here, the gray scale map is furthermore nonlinear to emphasize the increased values of $u$ in the tail of the waves. The parameters are $\alpha=1, \beta=0.2, \kappa=1, \tau_{u}=0.1, \tau_{v}=1, \tau_{s}=0.1, l_{u}=0.001$, and $l_{s}=0.017$. The simulated (displayed) system size is $L=1.0(\Delta L=0.3)$, and the shown time interval in (c), (d) is $\Delta t=5$. In all space-time diagrams, space is displayed vertically and time horizontally.

component dynamics without stable pacemakers is recovered. The larger $\kappa$, the larger is the decrease of the inhibitor $v$ if $s>u$ and consequently the larger the activatory effect of $s$. For $l_{s} \rightarrow l_{u}$, the variable $s$ mimicks the variable $u$ and also no pacemakers are observed. If $l_{s}>l_{u}$, the pulses have a broader distribution in $s$ than in $u$ and hence we have locations in space where $s>u$. If we decrease $\beta$ and approach (and finally reach) the oscillatory regime, tails of pulses become more and more oscillatory and it becomes easier to produce pacemakers. Increasing $\beta$ shrinks the set of initial conditions yielding pacemakers. The results can be summarized in the following way: If we increase $\beta$, decrease $\kappa$, or decrease $l_{s}$, it becomes increasingly difficult to create or maintain a self-organized wave source. We thus can infer that proximity to the oscillatory regime, sufficiently strong diffusion of $s$, and sufficiently strong coupling strength are favorable for the formation of stable pacemakers.

Figure 2 displays the evolution of a system with pacemaker for a longer time interval. Since periodic boundary conditions have been applied, not only the pacemaker is observed (in the upper part of the figure), but also the location where the waves collide (in the lower part of the figure). As already noticed, the first waves emitted by the pacemaker strongly resemble pulses. Later, the wave number and the frequency of the waves increase with time. However, the pulselike waves emitted first are faster than the subsequent ones; i.e., the wave speed decreases with time. As indicated by the different gray levels for $s$ in Fig. 2(a), the maximum value of $s$ within a pulse-like wave is significantly larger than in those waves which form the asymptotic wave train. Furthermore, the amplitude of $s$ within the collision zone of the waves is also much larger than within the wave train. The difference in amplitude between the first (pulselike) waves and the asymptotic wave train is restricted to the variable $s$. The values of $u$ in a pulse and in the asymptotic wave train are very similar [Fig. 2(b)]. In the asymptotic regime, the wave number of the propagating waves is constant. Within
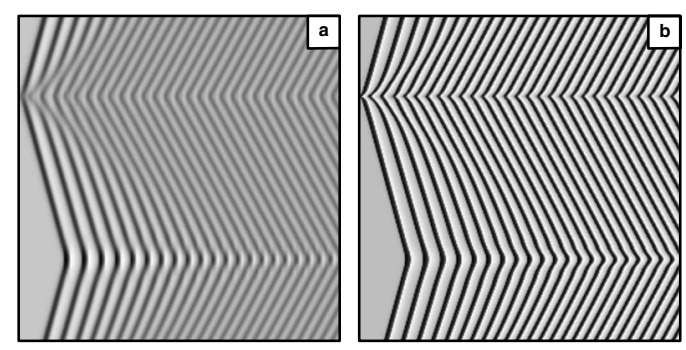

FIG. 2. Stable self-organized pacemaker in one spatial dimension. Space-time diagrams for (a) $s$ and (b) $u$ are shown. The parameters are $\alpha=1, \quad \beta=0.2, \quad \kappa=1, \tau_{u}=0.05, \tau_{v}=1, \tau_{s}=0.1, l_{u}$ $=0.00071$, and $l_{s}=0.017$. The displayed time interval is $\Delta t=50$, and the system size is $L=1$. Periodic boundary conditions are used. 


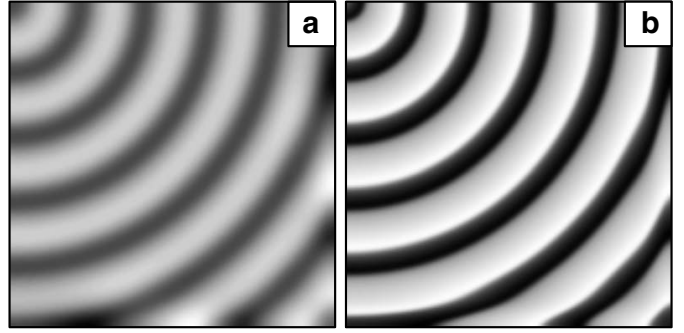

FIG. 3. Stable self-organized pacemaker in two spatial dimensions. Snapshots of the distributions of (a) $s$ and (b) $u$ are displayed. The parameters are $\alpha=1, \beta=0.2, \kappa=1, \tau_{u}=0.1, \tau_{v}=1, \tau_{s}=0.1, l_{u}$ $=0.05, l_{s}=1, L_{x}=L_{y}=15$, and $t=500$. No-flux boundary conditions are used.

the range of perturbations that lead to pacemakers, no dependence of the asymptotic wave number on the specific width of perturbation is detected. If a wave train created by a pacemaker is isolated from the pacemaker and forced to travel in the medium with periodic boundary conditions, we can check the stability of the wave train. It is possible to delete single waves from the train without observing a reappearance of the waves. Thus, pulse trains with different wavelengths are admitted solutions and we demonstrate that the asymptotic wavelength is selected by the pacemaker.

Simulations of two-dimensional systems have also been performed. Figure 3 shows an example of a target wave pattern present in a system with no-flux boundary conditions. The perturbation has been applied in a corner of the system to show as many waves as possible. In Fig. 3(a), the variable $s$ is shown. As with the one-dimensional system, the variable $s$ has an intermediate amplitude within the wave train and a large amplitude where the waves collide (here with the noflux boundary). Figure 3(b) shows that the amplitude of the activator $u$ does not undergo any significant changes at the boundary. Performing several two-dimensional simulations, we find that the evolution of that pattern is qualitatively similar to the one-dimensional case; i.e., a pacemaker arises only for appropriate perturbations and the amplitude of $s$ in the asymptotic pattern is smaller than in the initial phase of the dynamics.

\section{BISTABILITY OF PULSE SOLUTIONS}

In the previous section, it has been shown that stable selforganized pacemakers can appear in system (1). In this section, we show that the system also admits two stable types of pulse solutions. Figure 4 shows the profiles of three different patterns found in one-dimensional systems: a target pattern with pacemaker [(a)], a small pulse [(b), left], and a large pulse [(b), right]. All these patterns are stable with respect to small spatiotemporal perturbations.

The large pulse corresponds to the pulse already seen in Fig. 1. It is created by applying a sufficiently large perturbation of $u$ to the uniform steady state. Its velocity is the largest among the velocities of the three solutions, and the pattern is characterized by the largest amplitude of $s$ within the pulse. In this pulse solution, the property of $s$ acting as a second activator, leading to a large deviation of both $u$ and $s$ from
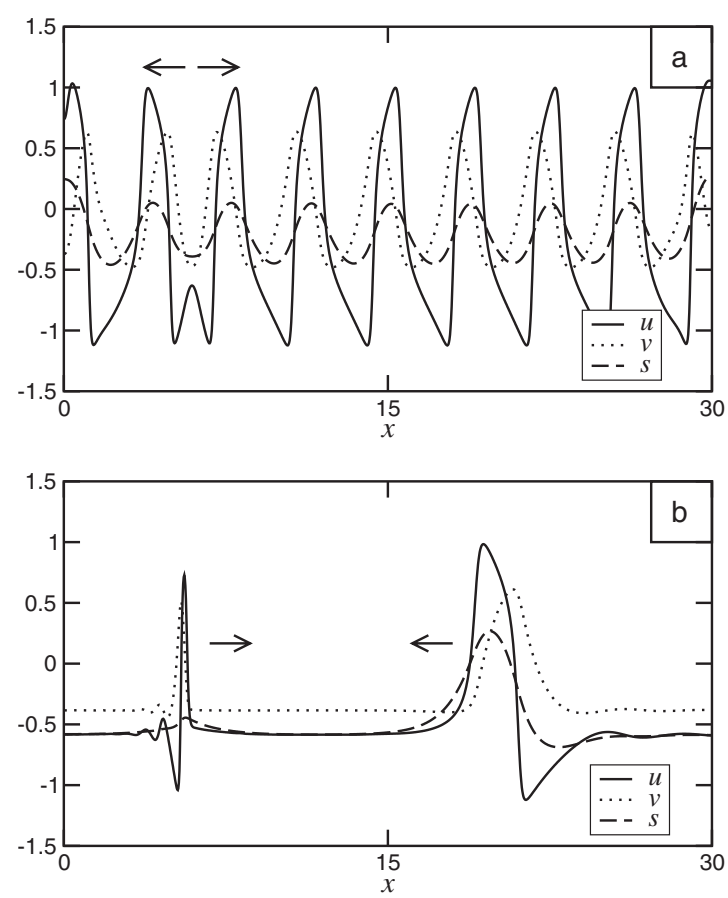

FIG. 4. Pacemaker and pulse solutions. The profiles of the variables are shown for a pacemaker (a) and the two pulse solutions (b). The parameters are $\alpha=1, \beta=0.2, \kappa=1, \tau_{u}=0.1, \tau_{v}=1, \tau_{s}=0.1, l_{u}$ $=0.05, l_{s}=1$, and $L=30$. The solid, dotted, and dashed lines correspond to $u, v$, and $s$, respectively. No-flux boundary conditions are present in (a), being responsible for the increase of $s$ at the boundary. The arrows indicate the direction of wave propagation.

their stationary values, is clearly seen. If this pulse solution is slightly perturbed, the perturbation is damped out quickly. Simulations indicate that this also holds for relatively strong perturbations, showing that this pulse solution is robust.

The small pulse is closely related to the pulse solution known for the reduced model without $s$. It can be obtained by performing a simulation starting with $\kappa=0$ and then slowly increasing $\kappa$ without destroying the pulse (here, to $\kappa=1$ ). This pulse solution is not only short, but also slow compared to the large pulse solution. The distribution of $s$ within the pulse only shows a small deviation from the stationary value. This pulse solution is also stable with respect to small perturbations. However, it can easily be destabilized with moderate perturbations, typically giving rise to a large pulse, although pacemakers are also created occasionally.

The target pattern is formed by a wave train with a wave speed which lies between the velocities of the two pulse solutions. The amplitude of $s$ within the wave train-i.e., the difference between the maximum and minimum values of $s$-also lies between the large amplitude for the large pulse and the small amplitude for the small pulse. In order to compare the widths of the pulses with the width of a single wave in the target pattern, it is convenient to consider the halfwidth of a wave as the distance between the maximum and minimum of the profile of the activator $u$. In the case of the wave train, the half-width is approximately half of one wavelength. The half-width of the target wave lies in between the half-widths of the two pulses.

For the set of parameters $\alpha=1, \beta=0.2, \tau_{u}=\tau_{s}=0.1, \tau_{v}=1$, $l_{u}=0.05$, and $l_{s}=1$, bistability of pulses is observed for 

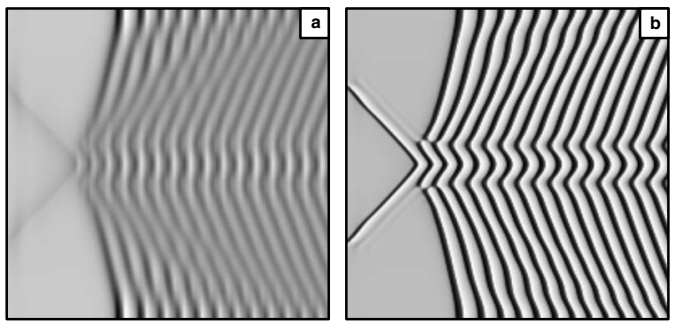

FIG. 5. Two colliding small pulses lead to a bound state of two pacemakers and a wave sink. Space-time diagrams for (a) $s$ and (b) $u$. The parameters are $\alpha=1, \beta=0.2, \kappa=1, \tau_{u}=0.1, \tau_{v}=1, \tau_{s}=0.1$, $l_{u}=0.05$, and $l_{s}=1$. The displayed time interval is $\Delta t=40$, and the system size is $L=20$. No-flux boundary conditions are used.

$0.73<\kappa<1.09$. For $\kappa<0.73$, only the small pulse is stable, while for $\kappa>1.09$ only the large pulse is stable (larger values than $\kappa=1.2$ have not been considered in the simulations).

\section{INTERACTION OF PULSES AND PACEMAKERS}

The two different kinds of pulse solutions are stable with respect to small perturbations and travel persistently on the background of the steady state in a one-dimensional medium with periodic boundary conditions. However, when pulses of both types are present in such a medium, they necessarily interact because they travel with different velocities. Furthermore, pulses of the same kind may collide and give rise to complex spatiotemporal patterns. The following interactions have been studied for a fixed set of parameters: large pulse, large pulse; large pulse, waves from a pacemaker; small pulse, small pulse; large pulse, small pulse (head-on and the large pulse running into the back of the small one); and small pulse, waves from a pacemaker (head-on and the target waves running into the back of the small pulse).

When two large pulses meet, they annihilate in the collision and the system returns to the stationary state. This corresponds to the typical behavior of pulses in excitable media and is therefore not further discussed here. If a large pulse collides with waves emitted by a pacemaker, the large pulse is annihilated and the pacemaker entrains the medium.

At the location where two small pulses collide (Fig. 5), the amplitude of $s$ increases strongly, as displayed in Fig. 5(a). Subsequently, slightly apart from the collision zone, two symmetrically shifted pacemakers appear which form a

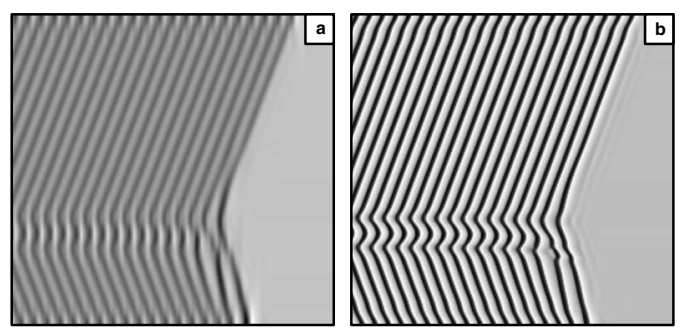

FIG. 6. Unstable bound state of pacemakers. Space-time diagrams for (a) $s$ and (b) $u$ are shown. The parameters are like in Fig. 5. The displayed time interval is $\Delta t=50$, and the system size is $L$ $=25$. No-flux boundary conditions are imposed.

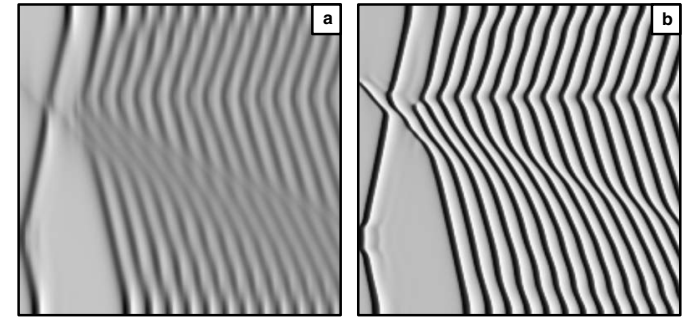

FIG. 7. Interaction of a small pulse (coming from above) with a large pulse (coming from below). A stable pacemaker is formed. Space-time diagrams for (a) $s$ and (b) $u$ for the initial evolution of the system are shown. The parameters of the system are as in Fig. 5.

bound state. Between the two wave sources, there is a small region where the waves collide and where the component $s$ performs large-amplitude oscillations. The frequency and wave number of the waves emitted by such a bound state of two pacemakers are larger than the corresponding values of the waves emitted by only one pacemaker. The bound state of two pacemakers emits hundreds of waves, but it is actually found to be unstable for the studied sets of parameters and the system finally returns to the rest state. This is shown for a different simulation in Fig. 6.

If a large and a small pulse interact, as displayed in Fig. 7, the waves first seem to annihilate. However, the large pulse actually reappears and proceeds into its initial direction. Also the small pulse reappears and first seems to proceed as before. Yet it becomes unstable and transforms into a large pulse. Then, in the tail of this pulse, a pacemaker appears. In the wave pattern subsequently formed, the impact of the small pulse is still seen as a local modulation of $s$ which is advected toward the border and finally decays there. The asymptotic state consists of a stable pacemaker located close to the collision zone of the pulses.

If the different pulses do not meet in a head-on collision, but if the fast (large) pulse runs into the back of the slow (short) one, the asymptotic state also consists of a pacemaker that is created at the location of the collision. Corresponding simulations are not shown here.

The next interaction scenario is the case of a small pulse colliding with the waves emitted by a pacemaker. The result of such an interaction is shown in Fig. 8. Within the collision zone, large-amplitude oscillations of $s$ appear, forming a localized pattern which can be clearly identified. This localized pattern can be interpreted as a disruption of the wave train of
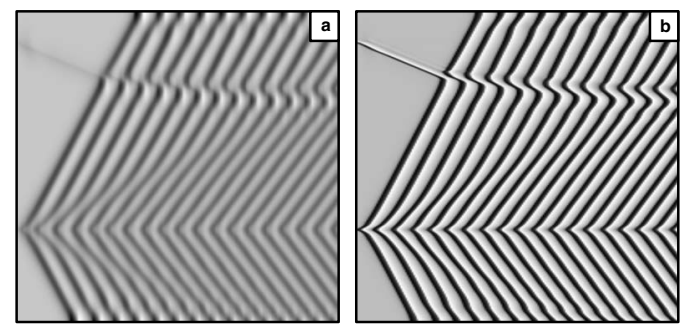

FIG. 8. Interaction of a small pulse with a pacemaker. Spacetime diagrams for the initial evolution of (a) $s$ and (b) $u$ are displayed. The parameters of the system are as in Fig. 5, except the system size, which is $L=50$. 


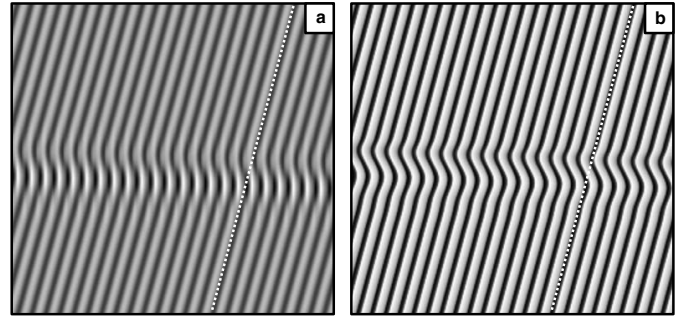

FIG. 9. Traveling phase flip. Space-time diagrams for (a) $s$ and (b) $u$ are shown. The parameters of the system are as in Fig. 5, except the system size $(L=22.625)$ and the displayed time interval $(\Delta t=50)$. Periodic boundary conditions are used.

the pacemaker. This pattern is not stationary, but travels at very low speed toward the original pacemaker. Finally, the traveling-localized pattern reaches the initial pacemaker and forms the type of bound state of two pacemakers already discussed in Fig. 5. This pattern is unstable, the pacemaker is finally destroyed, and the stationary state is recovered (cf. Fig. 6). The transient, however, is long and comprises hundreds of wave emissions.

The traveling-localized pattern can be interpreted as a moving phase flip on the background of traveling waves. The dynamics of this phase flip can be studied without the initial pacemaker in a one-dimensional system with periodic boundary conditions. This is shown in Fig. 9. The flip drifts at a low constant speed through the medium. The phase difference between the waves on the left and the right of the flip is $2 \pi$ (illustrated by the white dotted lines).

The last interaction scenario that has been studied consists of waves emitted by a pacemaker which run into the tail of the small pulse. Simulations not displayed here show that the waves from the pacemaker overtake the pulse and entrain the rest of the system. This means that the asymptotic state consists of the initial pacemaker. The small pulse produces a local perturbation which is advected to the no-flux boundary and decays there.

\section{DISCUSSION}

The two main results of this article are the observation of stable self-organized pacemakers in an excitable mediumi.e., not in an oscillatory medium-and the simultaneous existence of two stable pulse solutions. These three solutions allow the system to have a rich repertoire of spatiotemporal patterns, for which we can only give account of the most important ones. In the following, we will discuss the model, the most important findings, and their implications.

The system consists of a generic two-component activator-inhibitor system extended by an additional diffusing variable $s$. This component obeys linear kinetics and has been introduced to extend the two-component model in a simple way. The following conditions are sufficient to create a stable self-organized pacemaker: proximity to the regime of relaxational oscillations, sufficiently strong coupling of $s$ to the inhibitor $v$, and strong diffusion of the variable $s$ (compared to diffusion of $u$ ). Then, whether a pacemaker or a pair of pulses develops is a matter of the initial condition. Essen- tial for pacemaker formation is to provide a superthreshold perturbation for the activator $u$. This is accomplished by the variable $s$ : wherever $s>u, s$ decreases $v$ and hence favors the increase of $u$. Besides the coupling constant $\kappa$, which is responsible for the above-mentioned decrease of $v$ locally, the diffusion length $l_{s}$ must be larger than $l_{u}$ in order to establish a difference of $s$ and $u$ in the spatially extended system. To be more specific, in the example discussed in Sec. III, the inhibitor $v$ is decreased sufficiently in the space between the two tails of the first pulse pair emitted, such that in the center the increase of $u$ is possible. It is helpful to be close to the oscillatory regime where pulse tails tend to be nonmonotonous.

If the parameter $\beta$ is decreased slightly-such that the system is in the regime of relaxational oscillations - the formation of self-organized pacemakers is also observed. Thus, the mechanism leading to these structures is not restricted to the excitable regime. The wavelength of the target pattern is selected by the pacemaker. Therefore, this system constitutes an example of a three-component reaction-diffusion system showing stable autonomous pacemakers (for other examples see, e.g., Refs. [11,12] and references therein). An alternative way to create target patterns in excitable systems also in the absence of heterogeneities is the application of noise [19].

The model studied here consists of three independent variables. Several years ago, a two-component model with coexistence of excitable kinetics and stable uniform oscillations was presented [20,21]. There, self-organized pacemakers were also created by appropriate initial conditions. However, the pacemakers were unstable and uniform oscillations were approached asymptotically. Two main differences between that system and our model are that the local dynamics of our system is not oscillatory and the pacemakers studied here do not decay to uniform oscillations, but remain stable.

Within the last decades, it has been shown that reactiondiffusion systems can display interesting dynamics which cannot be satisfactorily described by standard twocomponent models, such as traveling and complex interacting spots, autonomous pacemakers, or complex pulse dynamics. In recent years, many articles investigated pattern formation in three-component reaction-diffusion systems: among the topics studied range the dynamics of spot solutions [15,16], birhythmicity [14], and front and pulse dynamics $[17,18,22]$. Particularly interesting in our context are Ref. [18] for studying heterogeneity-induced pulse dynamics, among them periodic wave emission (see also Ref. [22]), and Ref. [17] for observing the creation of a wave source as a result of the collision of pulses. The systems studied in those references have two inhibitors and model a planar dc gasdischarge system [23]. The three-component model studied here is not designed to explain the behavior of a specific reaction-diffusion system. The additional component $s$ inhibits the inhibitor $v$ and hence can be interpreted as mimicking the role of a second activator.

Complex pulse and spot interactions have been studied intensively and can also be found in two-component models. For work in this context, we refer to the work by Argentina et al. who showed that the transition from annihilation to preservation of colliding waves can proceed via a homoclinic bifurcation $[24,25]$ and to the work by Nishiura et al. who 
interpreted the interaction process in terms of unstable solutions called scattors [26].

In certain limit cases, three-component reaction-diffusion systems are essentially equivalent to two-component reaction-diffusion systems with nonlocal coupling [27]. A particular interesting effect of nonlocality is the emergence of multiscaled turbulence [28-30]. As long as we have two diffusive species in our system, no such turbulence is observed. However, simulations not shown here demonstrate that in the absence of diffusion of $u$, target patterns with turbulent and small-scale waves may develop. Also other recent work elucidates the influence of global and nonlocal coupling on pattern formation-e.g., drifting patterns [31], complex wave dynamics (among them also one-dimensional symmetric and asymmentric target patterns) in an electrochemical system [32], and the formation of bound states of pulses in excitable media [33]. Target patterns appearing for systems with global and long-range interaction have also been reported before [34,35].

Besides the solution corresponding to a pacemaker, also pulse solutions in the excitable regime of the full threecomponent model have been presented. For the studied sets of parameters, two stable types of pulses have been found, one resembling the pulse solution for the reduced twocomponent model, the other being a genuine threecomponent pulse solution with considerably larger velocity, amplitude, and width. Bistability of pulse solutions is considered to be relatively uncommon to reaction-diffusion systems. Winfree showed that multiple-spiral-wave solutions are possible in an excitable system $[36,37]$. There, the dispersion relation of the waves-i.e., the dependence of the velocity on the wavelength-shows an oscillatory behavior. The local minima of the dispersion relation determine the stable spiral solutions. Consequently, several spirals with different wavelengths and speeds may coexist in such a system. Another type of bistability of pulse solutions was presented by Bord- yugov and Engel $[38,39]$. There, pulse trains with identical wavelengths and different velocities may coexist. Here, the pulse solutions are very different in wavelengths and speed. While one pulse corresponds to the pulse solution known for reduced system, the other is genuine for the whole, coupled system and exists only for sufficiently large coupling strengths $\kappa$. We have focused on the formation of pacemakers, but a more detailed characterization of the pulse solutions in the three-component model is certainly an interesting topic for further study.

As a result of the different types of interaction between the pulses and pacemakers, not only may stable pacemakers appear, but also localized patterns representing bound states of pacemakers and traveling phase flips. We expect that this is just a first glance of the richness of possible wave patterns appearing in this three-component reaction-diffusion system. Here we emphasize that the appearance of pacemakers through pulse interaction demonstrates that the class of initial conditions giving rise to pacemakers is not small. Although the bound state of pacemakers is unstable in the simulations, its transient is long. Therefore, we cannot exclude that this pattern may be stable for other sets of parameters and may also have long-lasting counterparts in experiments, in particular in strictly one-dimensional systemse.g., electrochemical pattern formation on ring electrodes $[7,40]$. Since the bound state of two pacemakers emits waves with a larger frequency than one pacemaker only, the competition and interaction of pacemakers may be studied. Finding a stable bound state of pacemakers would mean that this pattern itself could be interpreted as a self-organized wave source and hence bistability of different pacemaker solutions could be possible.

\section{ACKNOWLEDGMENT}

The authors gratefully acknowledge discussions with $\mathrm{T}$. Mizuguchi.
[1] Y. Kuramoto, Chemical Oscillations, Waves, and Turbulence (Springer, Berlin, 1984).

[2] Chemical Waves and Patterns, edited by R. Kapral and K. Showalter (Kluwer Academic, Dordrecht, 1995).

[3] A. N. Zaikin and A. M. Zhabotinsky, Nature (London) 225, 535 (1970).

[4] S. Jakubith, H. H. Rotermund, W. Engel, A. von Oertzen, and G. Ertl, Phys. Rev. Lett. 65, 3013 (1990).

[5] S. Nasuno, M. Sano, and Y. Sawada, J. Phys. Soc. Jpn. 58, 1875 (1989).

[6] G. Gerisch, Wilhelm Roux' Arch. Entwickl.-Mech. Org. 156, 127 (1965).

[7] J. Christoph et al., J. Chem. Phys. 110, 8614 (1999).

[8] M. Stich and A. S. Mikhailov, Physica D 215, 38 (2006).

[9] C. Vidal and A. Pagola, J. Phys. Chem. 93, 2711 (1989).

[10] K. J. Lee, R. E. Goldstein, and E. C. Cox, Phys. Rev. Lett. 87, 068101 (2001).

[11] A. S. Mikhailov, Physica D 55, 99 (1992).

[12] M. Stich, M. Ipsen, and A. S. Mikhailov, Physica D 171, 19
(2002).

[13] H. Sakaguchi, Prog. Theor. Phys. 87, 241 (1992).

[14] V. Casagrande and A. S. Mikhailov, Physica D 205, 154 (2005).

[15] Y. Nishiura, T. Teramoto, and K.-I. Ueda, Chaos 15, 047509 (2005).

[16] S. V. Gurevich, S. Amiranashvili, and H.-G. Purwins, Phys. Rev. E 74, 066201 (2006).

[17] E. P. Zemskov, Phys. Rev. E 73, 046127 (2006).

[18] X. Yuan, T. Teramoto, and Y. Nishiura, Phys. Rev. E 75, 036220 (2007).

[19] S. Alonso, I. Sendina-Nadal, V. Perez-Munuzuri, J. M. Sancho, and F. Sagues, Phys. Rev. Lett. 87, 078302 (2001).

[20] R. Kobayashi, T. Ohta, and Y. Hayase, Phys. Rev. E 50, R3291 (1994).

[21] T. Ohta, Y. Hayase, and R. Kobayashi, Phys. Rev. E 54, 6074 (1996).

[22] A. Prat, Y.-X. Li, and P. Bressloff, Physica D 202, 177 (2005).

[23] C. P. Schenk, M. Or-Guil, M. Bode, and H.-G. Purwins, Phys. 
Rev. Lett. 78, 3781 (1997).

[24] M. Argentina, P. Coullet, and L. Mahadevan, Phys. Rev. Lett. 79, 2803 (1997).

[25] M. Argentina, P. Coullet, and V. Krinsky, J. Theor. Biol. 205, 47 (2000).

[26] Y. Nishiura, T. Teramoto, and K.-I. Ueda, Chaos 13, 962 (2003).

[27] Y. Kuramoto, D. Battogtokh, and H. Nakao, Phys. Rev. Lett. 81, 3543 (1998).

[28] Y. Kuramoto, Prog. Theor. Phys. 94, 321 (1995).

[29] Y. Kuramoto, H. Nakao, and D. Battogtokh, Physica A 288, 244 (2000)

[30] D. Battogtokh and Y. Kuramoto, Phys. Rev. E 61, 3227 (2000).
[31] E. M. Nicola, M. Or-Guil, W. Wolf, and M. Bär, Phys. Rev. E 65, 055101(R) (2002).

[32] F. Plenge, H. Varela, and K. Krischer, Phys. Rev. E 72, 066211 (2005).

[33] G. Bordyugov and H. Engel, Phys. Rev. E 74, 016205 (2006).

[34] U. Middya and D. Luss, J. Chem. Phys. 102, 5029 (1995).

[35] O. Nekhamkina and M. Sheintuch, Physica A 249, 134 (1998).

[36] A. T. Winfree, Phys. Lett. A 149, 203 (1990).

[37] A. T. Winfree, Physica D 49, 125 (1991).

[38] G. Bordiougov and H. Engel, Phys. Rev. Lett. 90, 148302 (2003).

[39] G. Bordyugov and H. Engel, Chaos 18, 026104 (2008).

[40] P. Grauel, H. Varela, and K. Krischer, Faraday Discuss. 120, 165 (2002). 